\title{
Gravitational Wave Production by Collisions: More Bubbles
}

\author{
Stephan J. Huber ${ }^{(1)}$ and Thomas Konstandin ${ }^{(2)} *$ \\ (1) Department of Physics and Astronomy, \\ Sussex, Falmer, Brighton, BN1 9QH, UK and \\ (2) IFAE, Universitat Autònoma de Barcelona, E-08193 Bellaterra, Barcelona, Spain
}

(Dated: October 30, 2018)

\begin{abstract}
We reexamine the production of gravitational waves by bubble collisions during a first-order phase transition. The spectrum of the gravitational radiation is determined by numerical simulations using the "envelope approximation". We find that the spectrum rises as $f^{3.0}$ for small frequencies and decreases as $f^{-1.0}$ for high frequencies. Thus, the fall-off at high frequencies is significantly slower than previously stated in the literature. This result has direct impact on detection prospects for gravity waves originating from a strong first-order electroweak phase transition at space-based interferometers, such as LISA or BBO. In addition, we observe a slight dependence of the peak frequency on the bubble wall velocity.
\end{abstract}

\section{INTRODUCTION}

Colliding bubbles in a first-order phase transition constitute one possible source of stochastic gravitational wave (GW) radiation [1, 2]. If the electroweak phase transition is strongly first-order, for instance, the kinetic energy stored in the Higgs field and the bulk motion of the plasma is partially released into gravity waves. This happens mostly at the end of the phase transition, when collisions break the spherical symmetry of the individual Higgs field bubbles. This possibility was systematically analyzed in a series of papers [3, 4, 5, 6]. The first simulation [3] consisted hereby of the full scalar field dynamics of two bubbles in vacuum, where the essential observation was made that the emitted radiation depends only on the gross features of the problem, namely the kinetic energy stored in the uncollided bubble regions. This observation is the basis of the so-called envelope approximation that

*s.huber@sussex.ac.uk; konstand@ifae.es 
opened up the possibility of simulating phase transitions with a large number of bubbles. This was subsequently exploited in refs. [4, 5] and further refined for a thermal environment in ref. [6].

In the case of only two colliding bubbles, the spectrum decreases as $f^{-1.8}$ for high frequencies. But this result might be special to the case of two bubbles, where the collision never finishes, which makes the introduction of a time cutoff function mandatory [3]. The situation is different if a realistic phase transition with a large number of bubbles is simulated. There were hints in refs. [4, 5, 6] that the spectrum of multi-bubble simulations might be more flat than in the two bubble case, but the numerical accuracy prohibited a conclusive statement. As a result, the frequency fall-off of the two bubble case is still being used in the present day literature [7, 8, 9].

The aim of the present work is to reexamine the generated spectrum of GWs by simulating a phase transition with a large number of bubbles, making use of the aforementioned envelope approximation. Compared to ref. [6], the numerical accuracy will be considerably improved, and a larger portion of the spectrum will be determined to allow for a careful analysis of the high frequency behavior.

\section{DETERMINATION OF THE GW SPECTRUM}

The fundamental quantity that enters the determination of the gravitational radiation are the spatial components of the stress-energy tensor $T_{i j}(\mathbf{x}, t)$. For a thermal phase transition it consists of the scalar field part and the plasma contribution. The total energy radiated into a direction $\hat{\mathbf{k}}$ is then given by [10]

$$
\frac{d E_{G W}}{d \omega d \Omega}=2 G \omega^{2} \Lambda_{i j, l m}(\hat{\mathbf{k}}) T_{i j}^{*}(\hat{\mathbf{k}}, \omega) T_{l m}(\hat{\mathbf{k}}, \omega),
$$

where $T_{i j}(\hat{\mathbf{k}}, \omega)$ denotes the stress-energy tensor in Fourier space

$$
T_{i j}(\hat{\mathbf{k}}, \omega)=\frac{1}{2 \pi} \int d t e^{i \omega t} \int d^{3} x e^{-i \omega \hat{\mathbf{k}} \cdot \mathbf{x}} T_{i j}(\mathbf{x}, t),
$$

and $\Lambda$ is the projection tensor for the transverse-traceless part

$$
\Lambda_{i j, l m}(\hat{\mathbf{k}})=\delta_{i l} \delta_{j m}-2 \hat{\mathbf{k}}_{j} \hat{\mathbf{k}}_{m} \delta_{i l}+\frac{1}{2} \hat{\mathbf{k}}_{i} \hat{\mathbf{k}}_{j} \hat{\mathbf{k}}_{l} \hat{\mathbf{k}}_{m}-\frac{1}{2} \delta_{i j} \delta_{l m}+\frac{1}{2} \delta_{i j} \hat{\mathbf{k}}_{l} \hat{\mathbf{k}}_{m}+\frac{1}{2} \delta_{l m} \hat{\mathbf{k}}_{i} \hat{\mathbf{k}}_{j} .
$$

Eq. (11) is derived in the wave zone approximation that is well justified in the present case. 
The key idea of the envelope approximation [5] is that the GW production does not depend on the details of the evolution of the scalar field in the region of the collided bubbles, but rather on the gross features of the problem, namely the shape of the uncollided bubble walls. Denoting the times and positions of the nucleated bubbles by $t_{n}$ and $\mathbf{x}_{n}$, this turns eq. (2) into

$$
T_{i j}(\hat{\mathbf{k}}, \omega)=\frac{1}{2 \pi} \int d t e^{i \omega t} \sum_{n} \int_{S_{n}} d \Omega \int d r r^{2} e^{-i \omega \hat{\mathbf{k}} \cdot\left(\mathbf{x}_{n}+r \hat{\mathbf{x}}\right)} T_{i j, n}(r, t),
$$

where $S_{n}$ denotes the uncollided region of the $n$th bubble and $T_{i j, n}$ its stress-energy tensor. If the phase transition proceeds by detonation, the stress energy is concentrated in a shell of bulk motion that is thin compared to the bubble radius, thus motivating the thin-wall approximation [6]

$$
4 \pi \int d r r^{2} e^{-i \omega \hat{\mathbf{k}} \cdot\left(\mathbf{x}_{n}+r \hat{\mathbf{x}}\right)} T_{i j, n}(r, t) \approx \frac{4 \pi}{3} e^{-i \omega \hat{\mathbf{k}} \cdot\left(\mathbf{x}_{n}+R_{n} \hat{\mathbf{x}}\right)} \hat{\mathbf{x}}_{i} \hat{\mathbf{x}}_{j} R_{n}^{3} \kappa \rho_{v a c},
$$

with $R_{n}(t)$ the size of the $n$th bubble and $\rho_{v a c}$ the difference in energy density between the true and the false vacuum (we use this terminology even though in the presence of a thermal bath the latent heat is the relevant quantity).

The efficiency factor $\kappa$ determines how much of the vacuum energy is transformed into kinetic energy of the bulk fluid instead of reheating the plasma inside the bubble. This coefficient can be determined as a function of the parameter $\alpha$ that is defined as the ratio between the vacuum energy and the total energy stored in radiation [6]

$$
\kappa(\alpha)=\frac{1}{1+0.715 \alpha}\left[0.715 \alpha+\frac{4}{27} \sqrt{\frac{3 \alpha}{2}}\right], \quad \alpha=\frac{\rho_{v a c}}{\rho_{\text {rad }}} .
$$

The last ingredient from the phase transition is the velocity of the bubble wall $v_{b}$. For very strong phase transitions it is given by [11]

$$
v_{b}=\frac{\sqrt{1 / 3}+\sqrt{\alpha^{2}+2 \alpha / 3}}{1+\alpha} .
$$

In fact, eq. (77) is not true in general. In phase transitions, there exists a larger class of detonation solutions, as discussed in ref. [12]. Therefore eq. (77) gives only a lower bound on the wall velocity and we will treat $v_{b}$ as a free parameter in our analysis. 
Following the above considerations, eq. (4) can be rewritten as

$$
\begin{aligned}
T_{i j}(\hat{\mathbf{k}}, \omega) & =\kappa \rho_{v a c} v_{b}^{3} C_{i j}(\hat{\mathbf{k}}, \omega) \\
C_{i j}(\hat{\mathbf{k}}, \omega) & =\frac{1}{6 \pi} \sum_{n} \int d t e^{i \omega\left(t-\hat{\mathbf{k}} \cdot \mathbf{x}_{n}\right)}\left(t-t_{n}\right)^{3} A_{n, i j}(\hat{\mathbf{k}}, \omega), \\
A_{n, i j}(\hat{\mathbf{k}}, \omega) & =\int_{S_{n}} d \Omega e^{-i \omega v_{b}\left(t-t_{n}\right) \hat{\mathbf{k}} \cdot \hat{\mathbf{x}}} \hat{\mathbf{x}}_{i} \hat{\mathbf{x}}_{j} .
\end{aligned}
$$

An efficient way of evaluating these integrals numerically is presented in the Appendix.

Before we discuss the simulation of the phase transition, we comment on the so-called quadrupole approximation that is given by the limit $\mathbf{k} \cdot \mathbf{x} \rightarrow 0$ or

$$
C_{i j}(\omega)=\frac{1}{6 \pi} \sum_{n} \int d t e^{i \omega t}\left(t-t_{n}\right)^{3} A_{n, i j}(0, t) .
$$

This approximation becomes exact in the limit of small frequencies. For large frequencies, it turns out that this substitution in the case of two bubbles overestimates the resulting GW production by more than one order of magnitude; additionally, the peak frequency is shifted to larger values what was first noticed in the simulation of the scalar field evolution of two single bubbles in ref. [3]. This result is rather surprising, since e.g. in electrodynamics with a localized source, the quadrupole approximation generally underestimates the full result. However, in the present case the assumption of localized sources is not applicable such that the quadrupole approximation can overestimate the full result as detailed in ref. [3].

To model the phase transition, we assume that the nucleation probability per volume and time is given by

$$
P=P_{0} \exp \left(\beta\left(t-t_{0}\right)\right)
$$

The parameter $\beta^{-1}$ is approximately the duration of the phase transition. Since $\beta$ is the sole dimensionful parameter of the phase transition, the fraction of energy liberated into gravitational wave radiation per frequency octave is [6]

$$
\Omega_{G W *}=\omega \frac{d E_{G W}}{d \omega} \frac{1}{E_{t o t}}=\kappa^{2}\left(\frac{H}{\beta}\right)^{2}\left(\frac{\alpha}{\alpha+1}\right)^{2} \Delta\left(\omega / \beta, v_{b}\right),
$$

where we used the definition of the Hubble constant

$$
H^{2}=\frac{8 \pi G \rho_{t o t}}{3}=\frac{8 \pi G\left(\rho_{v a c}+\rho_{r a d}\right)}{3},
$$

and defined the dimensionless function $\Delta$ as

$$
\Delta\left(\omega / \beta, v_{b}\right)=\frac{\omega^{3}}{\beta^{3}} \frac{3 v_{b}^{6} \beta^{5}}{2 \pi V} \int d \hat{\mathbf{k}} \Lambda_{i j, l m} C_{i j}^{*} C_{l m} .
$$


If the integration is performed over a large volume $V$ of the Universe (in terms of $\beta^{-3}$ ) with $N$ bubbles, one obtains in the quadrupole approximation

$$
\int d \hat{\mathbf{k}} \Lambda_{i j, l m} C_{i j}^{*} C_{l m} \propto N \beta^{-8} \propto \frac{V}{v_{b}^{3} \beta^{5}},
$$

and hence we recover the well known result [6]

$$
\frac{E_{G W}}{E_{t o t}} \propto \kappa^{2} v_{b}^{3}\left(\frac{H}{\beta}\right)^{2}\left(\frac{\alpha}{\alpha+1}\right)^{2} .
$$

The remainder of the present work is devoted to discussing numerical results for the function $\Delta\left(x, v_{b}\right)$.

\section{NUMERICAL RESULTS}

To calculate the function $\Delta\left(x, v_{b}\right)$, we proceed as follows. First, we choose the main parameters of the simulation: The radius of the part of the Universe under consideration $L_{U}$, and the number of directions to integrate over, $N_{k}$. Since the GW production is isotropic, the directional variation indicates the statistical uncertainty of the simulation.

Using this information, we simulate a scenario with random nucleation times and locations for the bubbles following the nucleation probability in eq. (12). As in ref. [5], our volume is spherical and the bubbles are cut off by the boundary which is equivalent to having a mirror symmetric configuration beyond the volume.

The result for a small and a large Universe and a few directions, $N_{k}=32$, is shown in Fig. 1. For large wall velocities, the size of the simulated part of the Universe mostly influences the total amplitude of the GW radiation. However, for smaller wall velocities, the produced radiation at high frequencies depends crucially on the size of the Universe. For small velocities the high frequency part of the spectrum approaches the quadrupole approximation, since in this case $\mathbf{k} \cdot \mathbf{x} \rightarrow \mathbf{k} \cdot \mathbf{x}_{\mathbf{n}}$. This is not identical to the quadrupole approximation (only the phase in eq. (10) vanishes), but it turns out that the additional phase in eq. (9) from the bubble position solely leads to a slight suppression of the low frequency part of the spectrum in our numerical results.

The enhancement of the high-frequency part in the quadrupole approximation can be explained in the following way. After the nucleation of the first bubble, the phase transition ends when the whole space is in the new phase, which typically takes a time $\tau \approx 5 / \beta$. 

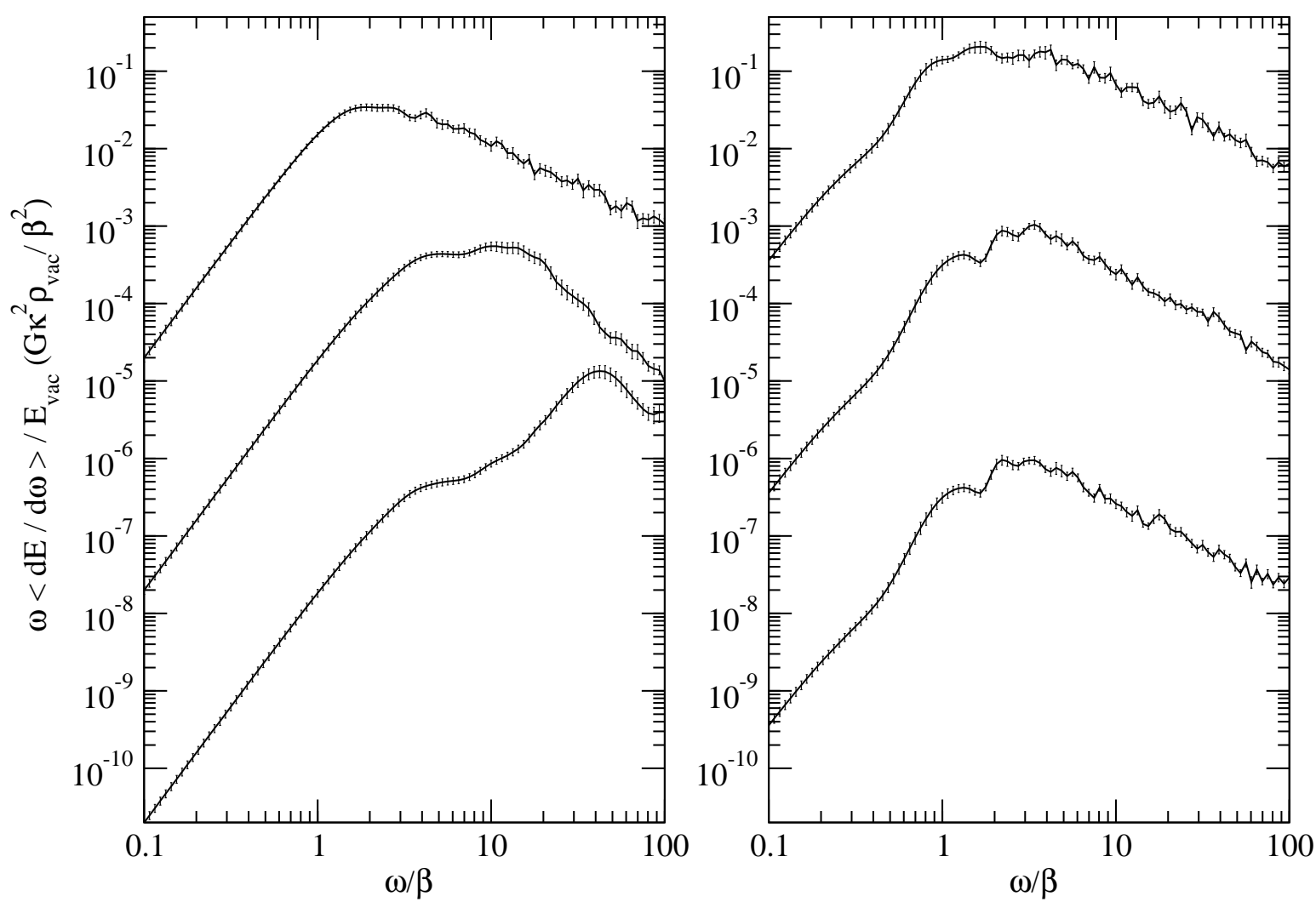

FIG. 1: The left panel shows the fraction of gravitational radiation for a small simulation, $L_{U}=$ $3 v_{b} / \beta, 7$ bubbles. For small wall velocities, significant boundary effects are visible. The right simulation is relatively large, $L_{U}=7 v_{b} / \beta, 109$ bubbles. Both simulations have been integrated over $N_{k}=32$ directions. Velocities decrease from top to bottom as $v_{b}=\{1,0.1,0.01\}$.

However, if the radius of the simulated volume $L_{U}$ is chosen too small, the simulation ends when the first nucleated bubble covers the whole spherical volume. This leads to a suppression of the total GW density and also to a rather steep edge in the time profile that amplifies the high frequency part of the spectrum. This enhancement effect is nonphysical and just arises because of the finite size of our test volume and the presence of the boundary. It disappears for larger volumes, when the phase transition is finished before the first nucleated bubble covers the whole spherical volume and boundary effects are small. For large bubble velocities, when the quadrupole approximation does not apply, the enhancement also disappears, since effects from large bubbles are in general reduced due to the fast oscillation of the integrand in eq. (10). This motivates our final choice $L_{U}=7 v_{b} / \beta$ in order to suppress these boundary effects.

The error bars in Fig. 1 result from the integration over the $N_{k}=32$ different directions. 
If the number of directions would be further increased, most of the displayed oscillatory features in the spectrum would persist, since they result from the finiteness of the size of the simulation $L_{U}$ and depend on the specific nucleation positions and times of the randomly generated scenario. In addition, the amplitude of the low frequency part of the spectrum and the total emitted radiation depend sensitively on the times of the first bubble nucleation and first collision, which are however quite similar in the different scenarios. To decrease these features, one has to further increase the size of the Universe and/or average over several scenarios. We will do the latter in the following.

Our final results, shown in Fig. 2, are obtained for the parameters

$$
L_{U}=7 v_{b} / \beta, \quad N_{k}=32,
$$

and averaged over eight scenarios. The given error bars result from this averaging procedure. For very small frequencies, one expects $\Omega_{G W *}$ to scale as $\omega^{3}$, since $C_{i j}(\omega)$ becomes constant in this limit. This is reflected by the numerical results. For large frequencies, we find that $\Omega_{G W *}$ scales as $\omega^{-1}$, unlike the two-bubbles case that was discussed in ref. [3]. We parametrize the spectrum as

$$
\Omega_{G W *}\left(f_{*}\right)=\tilde{\Omega}_{G W *} \frac{(a+b) \tilde{f}_{*}^{b} f_{*}^{a}}{b \tilde{f}_{*}^{(a+b)}+a f_{*}^{(a+b)}},
$$

with the peak frequency $\tilde{f}_{*}=\tilde{\omega} / 2 \pi$ and the peak amplitude $\tilde{\Omega}_{G W *}$ and both parameters are functions of the wall velocity. The two exponents obtained by fitting lie in the range $a \in[2.66,2.82]$ and $b \in[0.90,1.19]$. The most interesting case from a phenomenological point of view is given by large wall velocities, $v_{b} \approx 1$, in which case the fit yields $a \approx 2.8$ and $b \approx 1.0$. Note that the fit is optimized for a frequency range close to the peak frequency, and does not correctly reproduce the asymptotic low frequency behavior.

The main result of our analysis is that the spectrum scales for high frequencies close to $\omega^{-1}$ in contrast to the two bubble case presented in ref. [3]. This feature is already present for a rather small volume (just a few bubbles) and high wall velocities $\left(v_{b} \simeq 1\right.$ ) as depicted in the left panel of Fig. 1. The reason for this qualitative difference between the results is hence probably the time-dependent cutoff function that has been used in ref. [3] in order to terminate the phase transition.

The spectrum that would be observed today is obtained by red-shifting this result ac- 

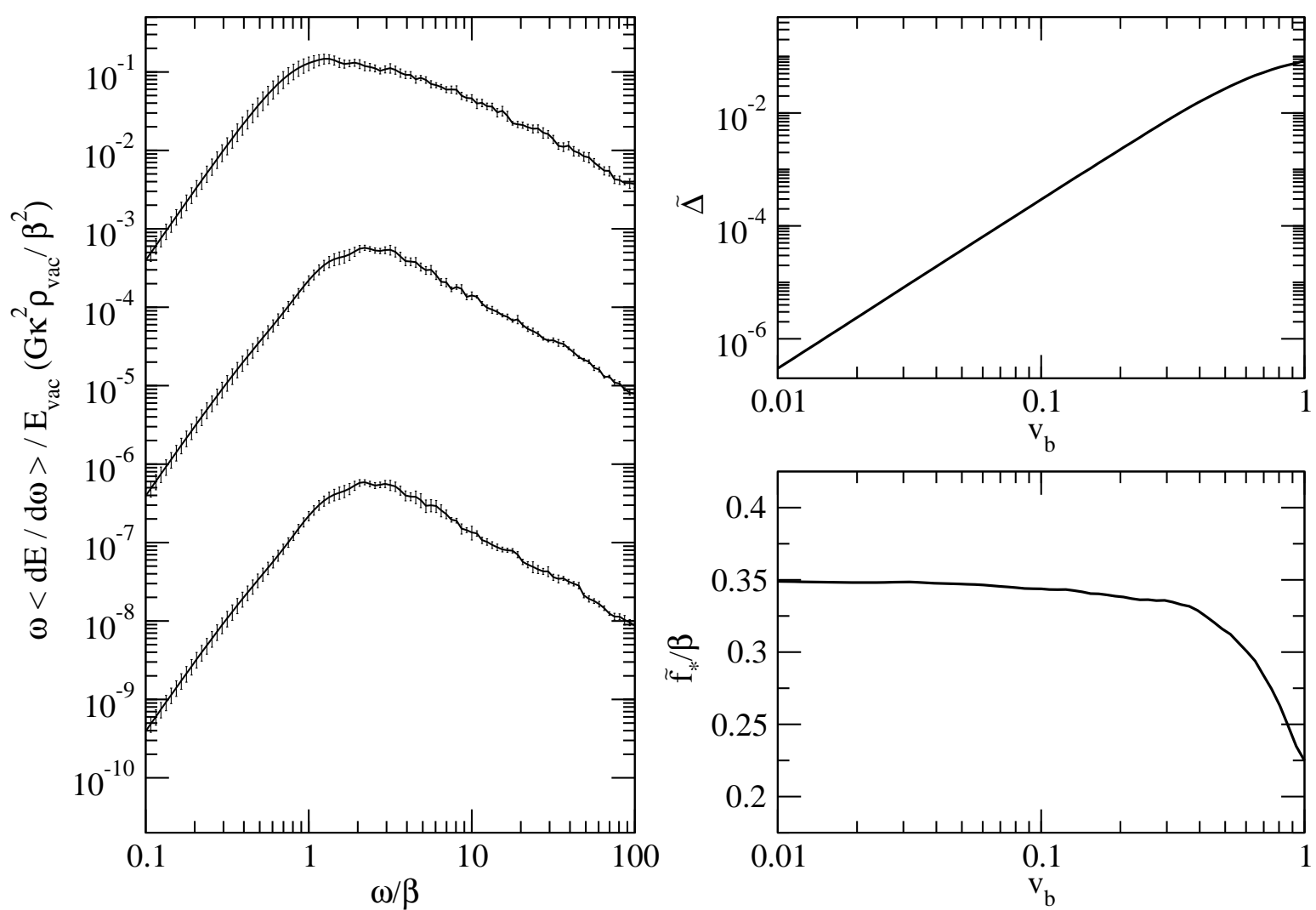

FIG. 2: The left panel shows the spectrum of gravitational radiation for a simulation with $L_{U}=$ $7 v_{b} / \beta$, integrated over $N_{k}=32$ directions. The error bars result from averaging over eight different scenarios. Velocities decrease from top to bottom as $v_{b}=\{1,0.1,0.01\}$. The right panel shows the parameters $\tilde{\Delta}$ and $\tilde{f}_{*} / \beta$ as functions of $v_{b}$.

cording to

$$
\begin{aligned}
\tilde{f} & =16.5 \times 10^{-3} \mathrm{mHz}\left(\frac{\tilde{f}_{*}}{\beta}\right)\left(\frac{\beta}{H_{*}}\right)\left(\frac{T_{*}}{100 \mathrm{GeV}}\right)\left(\frac{g_{*}}{100}\right)^{1 / 6}, \\
h^{2} \tilde{\Omega}_{G W} & =1.67 \times 10^{-5} \tilde{\Omega}_{G W *}\left(\frac{100}{g_{*}}\right)^{1 / 3} \\
& =1.67 \times 10^{-5} \tilde{\Delta} \kappa^{2}\left(\frac{H}{\beta}\right)^{2}\left(\frac{\alpha}{\alpha+1}\right)^{2}\left(\frac{100}{g_{*}}\right)^{1 / 3} .
\end{aligned}
$$

The two functions $\tilde{f}_{*} / \beta$ and $\tilde{\Delta}$ are displayed in the right panels of Fig. 2 and are approximately given by

$$
\begin{aligned}
\tilde{\Delta} & =\frac{0.11 v_{b}^{3}}{0.42+v_{b}^{2}}, \\
\tilde{f}_{*} / \beta & =\frac{0.62}{1.8-0.1 v_{b}+v_{b}^{2}} .
\end{aligned}
$$


Kosowsky et al

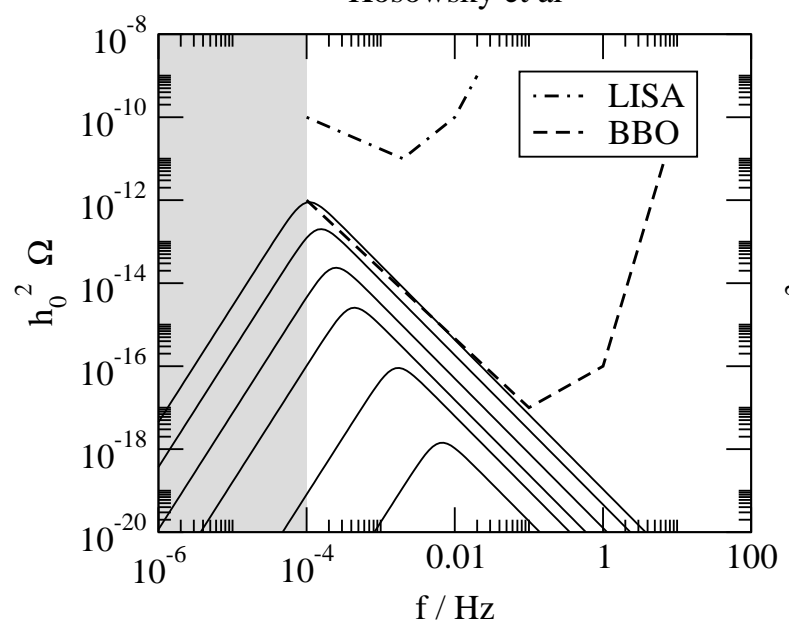

This work

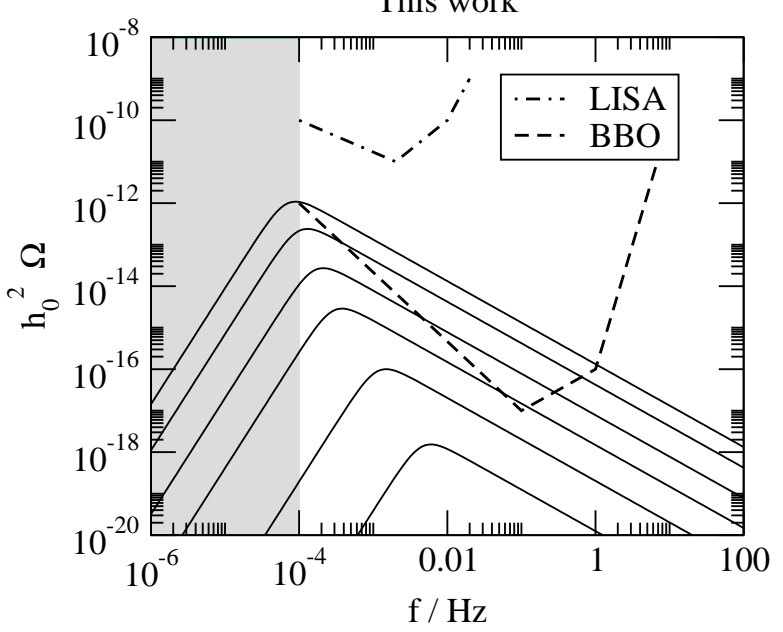

FIG. 3: Several spectra of gravitational radiation according to the old and new formulas. The parameters are taken from ref. [8] and given in table [ with $\alpha$ decreasing from top to bottom. In the shaded region, the sensitivity of LISA and BBO is expected to drop considerably.

Notice that the peak frequency and amplitude agree reasonably well with the results presented in ref. [6] (our peak amplitude is about 50\% larger for $v_{b} \approx 1$, while for small $v_{b}$ both results agree within the statistical errors). In the light of the analysis presented in ref. [13], the dependence of the peak frequency on the wall velocity has the following physical interpretation. For small velocities, $v_{b} \ll 1$, the phase transition lasts long compared to the relevant distance scale that is given by the average bubble size. In this case, the GW spectrum inherits the time scale of the source, $\tilde{f} \sim \beta$. If one used in eqs. (8) $-(10)$ a wall velocity much larger than the speed of light, $v_{b} \gg 1$, the phase transition would be very short compared to the relevant distance scale and the GWs would inherit the distance scale of the source, $\tilde{f} \sim \beta / v_{b}$. This effect leads to a decrease in the peak frequency in the transition region where the wall velocity is close to the speed of light.

\section{CONCLUSIONS}

We reexamined the spectrum of gravitational wave radiation generated by bubble collisions during a first-order phase transition in the envelope approximation. Using refined numerical simulations, our main finding is that the spectrum falls off only as $f^{-1.0}$ at high frequencies, considerably slower than appreciated in the literature. This behavior is most probably related to the many small bubbles nucleated at a later stage of the phase tran- 


\begin{tabular}{|c||c|c|c|}
\hline set & $\alpha$ & $\beta / H$ & $T_{*} / \mathrm{GeV}$ \\
\hline \hline 1 & 0.03 & 1000 & 130 \\
\hline 2 & 0.05 & 300 & 110 \\
\hline 3 & 0.07 & 100 & 85 \\
\hline 4 & 0.1 & 60 & 80 \\
\hline 5 & 0.15 & 40 & 75 \\
\hline 6 & 0.2 & 30 & 70 \\
\hline
\end{tabular}

TABLE I: Sets of parameters used in Fig. 3.

sition [32]. This result is especially interesting in the light of recent investigations [7, 8] that indicate that in the case of a first-order electroweak phase transition (obtained by a singlet sector [14, 15] or higher dimensional operators [16, 17, 18]) the peak frequency of the produced radiation is below the best sensitivity range of planed satellite experiments, such as LISA and BBO [19, 20]. This effect is shown in Fig. 3 for several typical parameter sets for the phase transition in the nMSSM [8]. Notice that the discussion in ref. [8] suggests that stronger phase transitions in general lead to smaller peak frequencies due to a decrease in the parameters $\beta / H$ and $T_{*}$. This amplifies the importance of the high frequency part of the gravitational wave spectrum. Notice also that a flatter spectrum simplifies the distinction from other sources of stochastic gravitational waves, such as turbulence [21, 22, 23, 24] or preheating after inflation [25, 26, 27, 28, 29, 30]. Besides, we found that the peak frequency slightly depends on the expansion velocity of the bubbles and decreases for higher wall velocities. Our quantitative results are summarized by eqs. (19)-(23).

Finally, we would like to comment on the recent paper [31], where an analytic approach to the GW production by collisions based on stochastic considerations was presented. In this approach, assumptions have to be made about the time-dependence of unequal time correlations of the velocity field. In their favored model, the authors obtain a scaling as $\omega^{-2}$ for the high frequency part of the spectrum. We suspect that this disparity is due to conceptual differences.

First, notice that the treatment presented here is based on two main ingredients: The thin wall and the envelope approximations. Even though the stochastic approach in ref. [31] does not require the thin wall approximation, the results are also valid in this limit, such that 
this approximation cannot be responsible for the different spectra. However, in the approach of ref. [31] the collided and uncollided regions of the bubbles are treated equally but in a stochastic manner. Breaking of the spherical symmetry, necessary for GW production, is encoded in assumptions on the velocity correlation functions. This is in contrast to our analysis in which the well tested [4] envelope approximation breaks the spherical symmetry in a realistic way.

Furthermore, only the time dependent mean bubble radius enters into the stochastic calculation, while in the analysis at hand bubbles with a realistic size distribution are simulated. The occurrence of many small bubbles probably enhances the high frequency part of the spectrum, as already argued in refs. [4, 5, 6].

Finally, it is interesting to see that even in the stochastic treatment the high frequency part of the spectrum can scale as $\omega^{-1}$ (in agreement with our results) if one assumes that the source is fully uncorrelated at unequal times. Especially at late stages of the phase transition, many small bubbles are generated, start to collide and are absorbed by neighboring bubbles at a large rate such that in this regime the assumption of non-correlation might indeed be plausible.

\section{Acknowledgments}

We would like to thank G. Servant for helpful discussions on ref. [31]. T.K. is supported by the Swedish Research Council (Vetenskapsrådet), Contract No. 621-2001-1611 and by the EU FP6 Marie Curie Research \& Training Network 'UniverseNet' (MRTN-CT-2006-035863).

\section{APPENDIX A: SOME DETAILS ABOUT THE NUMERICAL ALGORITHM}

In this section we will present an efficient way of evaluating the integrals given in eqs. (8))-

(10). It turns out that rotating the vector $\hat{\mathbf{k}}$ parallel to the $\mathrm{z}$-axis is very useful. One advantage is that the projection of the transverse-traceless part simplifies to

$$
\Lambda_{i j, l m} T_{i j}^{*} T_{l m}=\frac{1}{2}\left(T_{x x}^{*}-T_{y y}^{*}\right)\left(T_{x x}-T_{y y}\right)+T_{x y}^{*} T_{x y}+T_{y x}^{*} T_{y x}
$$




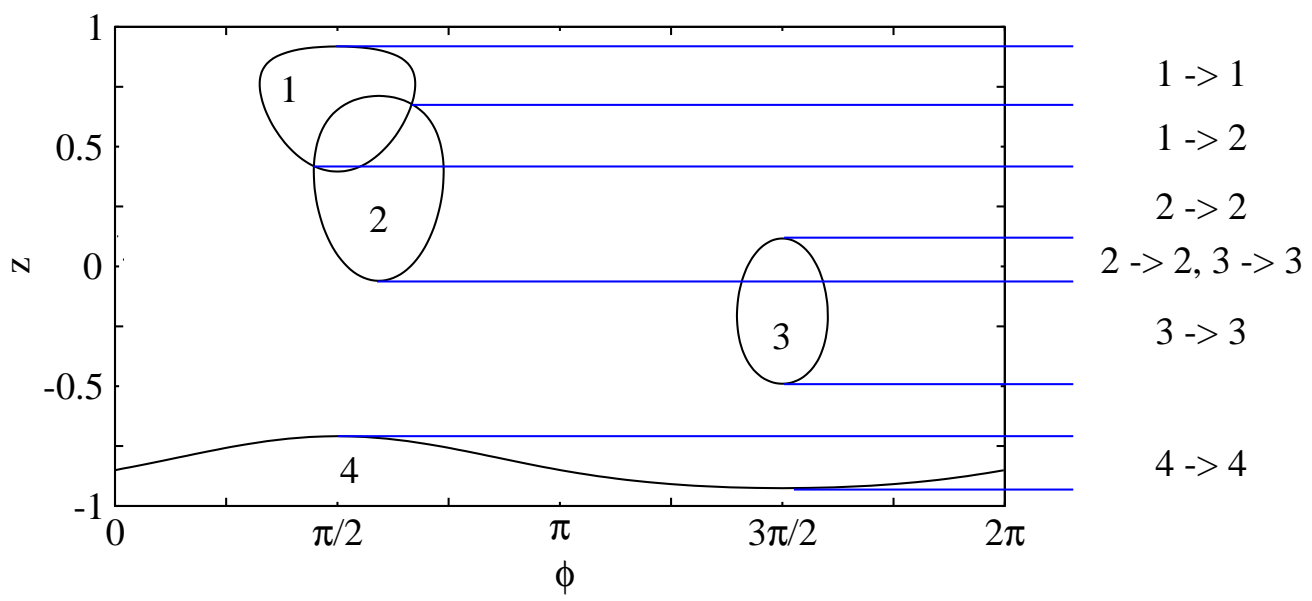

FIG. 4: The figure depicts the case of a bubble that intersects with four neighboring bubbles. The data structure we use contains the information which bubbles constitute the boundaries of the uncollided regions in each segment. This improves the performance greatly especially at the end of the phase transition when virtually all bubbles intersect with each other but only a few bubbles are relevant in each segment.

Using cylindrical coordinates and denoting the rotated uncollided region as $S_{n}^{\prime}$, this leads to

$$
\begin{gathered}
\frac{d E_{G W}}{d \omega d \Omega}=4 G \rho_{v a c}^{2} \kappa^{2} v_{b}^{6} \omega^{2}\left(\left|C_{+}\right|^{2}+\left|C_{-}\right|^{2}\right), \\
C_{ \pm}(\omega)=\frac{1}{6 \pi} \sum_{n} \int d t e^{i \omega\left(t-z_{n}\right)}\left(t-t_{n}\right)^{3} A_{n, \pm}(\omega, t), \\
A_{n, \pm}(\omega, t)=\int_{-1}^{1} d z e^{-i v_{b} \omega\left(t-t_{n}\right) z} B_{n, \pm}(z, t), \\
B_{n,+}(z, t)=\frac{\left(1-z^{2}\right)}{2} \int_{S_{n}^{\prime}} d \phi \cos (2 \phi), \quad B_{n,-}(z, t)=\frac{\left(1-z^{2}\right)}{2} \int_{S_{n}^{\prime}} d \phi \sin (2 \phi) .
\end{gathered}
$$

The second advantage of this coordinate system is that the integrals $B_{n, \pm}(z)$ do not depend on the frequency $\omega$ or the wall velocity $v_{b}$. Hence, the functions $B_{n, \pm}(z)$ need only to be determined once for all values of $\omega$ and $v_{b}$ what greatly improves the performance of the numerical evaluation of the integrals.

Notice that the function $A_{n, i j}(\omega, t)$ in eq. (A4) does not depend on the frequency $\omega$ and the wall velocity $v_{b}$ separately, but only on the product $\omega v_{b}$. Hence, we choose an exponential distribution for the values of the wall velocity $v_{b}$ and the frequency $\omega$ to reduce the number of necessary evaluations of $A_{n, \pm}(\omega, t)$. For each direction, we then evaluate the integrals (A2)-(A5) and integrate over the different directions. 
Finally, we would like to comment on the numerical accuracy of our simulation. The integration in eq. (A5) is performed by determining the intersections of the overlapping bubbles, while the integration in eq. (A4) is done on an adaptive grid such that the relative error never exceeds $10^{-5}$. This requires for the highest frequencies up to $10^{4}$ evaluations of the functions $B_{n, \pm}(z)$. In order to improve the performance we employ a data structure that for a fixed time $t$ and a specific bubble $n$ encodes the regions that are uncollided and do not intersect with other bubbles. The $z$-space is divided into different segments, where in every segment the data structure contains the information which bubbles constitutes the borders of the intersecting regions. A graphical representation of this data structure is depicted in Fig. 4. This is especially useful at late stages of the phase transition, when almost all bubbles intersect with each other, but only a few bubbles are relevant for each segment. The dominant error in the final spectrum results from the fact that the time integration in eq. (A3) is done on an equidistant grid with size $N_{t}=512$. We checked that the error resulting from this choice never exceeds a few percent for the presented results. As a first test, we used this algorithm to reproduce the results in the two bubble case and with a cutoff function as presented in ref. [5].

A final source of uncertainty is the finite size of the volume. As argued in the main text, the completion of the phase transition takes approximately the time $\tau \approx 5 / \beta$ and the the size of the volume under consideration should be chosen accordingly. It turns out that for the portion of the spectrum under consideration, a size of $L_{U}=7 v_{b} / \beta$ is sufficient to suppress boundary effects, as long as the first bubble nucleates not too far from the center of the volume which we assure in our simulations.

[1] E. Witten, "Cosmic Separation Of Phases," Phys. Rev. D 30 (1984) 272.

[2] C. Hogan, "Gravitational Radiation from Cosmological Phase Transitions," Mon. Not. R. Astron. Soc. 218 (1986) 629.

[3] A. Kosowsky, M. S. Turner and R. Watkins, "Gravitational Radiation From Colliding Vacuum Bubbles," Phys. Rev. D 45 (1992) 4514.

[4] A. Kosowsky, M. S. Turner and R. Watkins, "Gravitational waves from first order cosmological phase transitions," Phys. Rev. Lett. 69 (1992) 2026. 
[5] A. Kosowsky and M. S. Turner, "Gravitational Radiation From Colliding Vacuum Bubbles: Envelope Approximation To Many Bubble Collisions," Phys. Rev. D 47 (1993) 4372 arXiv:astro-ph/9211004.

[6] M. Kamionkowski, A. Kosowsky and M. S. Turner, "Gravitational radiation from first order phase transitions," Phys. Rev. D 49 (1994) 2837 arXiv:astro-ph/9310044.

[7] C. Grojean and G. Servant, "Gravitational waves from phase transitions at the electroweak scale and beyond," Phys. Rev. D 75 (2007) 043507 arXiv:hep-ph/0607107.

[8] S. J. Huber and T. Konstandin, "Production of Gravitational Waves in the nMSSM," JCAP 0805 (2008) 017 [arXiv:0709.2091 [hep-ph]].

[9] T. Kahniashvili, A. Kosowsky, G. Gogoberidze and Y. Maravin, "Detectability of Gravitational Waves from Phase Transitions," Phys. Rev. D 78 (2008) 043003 arXiv:0806.0293 [astro-ph]].

[10] S. Weinberg, "Gravitation and Cosmology," Wiley, New York (1982).

[11] P. J. Steinhardt, "Relativistic Detonation Waves And Bubble Growth In False Vacuum Decay," Phys. Rev. D 25, 2074 (1982).

[12] M. Laine, "Bubble Growth As A Detonation," Phys. Rev. D 49 (1994) 3847 arXiv:hep-ph/9309242.

[13] C. Caprini, R. Durrer and R. Sturani, "On the frequency of gravitational waves," Phys. Rev. D 74, 127501 (2006) arXiv:astro-ph/0607651.

[14] S. J. Huber and M. G. Schmidt, "Electroweak baryogenesis: Concrete in a SUSY model with a gauge singlet," Nucl. Phys. B 606 (2001) 183 [arXiv:hep-ph/0003122].

[15] J. R. Espinosa and M. Quiros, "Novel effects in electroweak breaking from a hidden sector," Phys. Rev. D 76 (2007) 076004 arXiv:hep-ph/0701145.

[16] C. Grojean, G. Servant and J. D. Wells, "First-order electroweak phase transition in the standard model with a low cutoff," Phys. Rev. D 71 (2005) 036001 arXiv:hep-ph/0407019].

[17] D. Bodeker, L. Fromme, S. J. Huber and M. Seniuch, "The baryon asymmetry in the standard model with a low cut-off," JHEP 0502, 026 (2005) arXiv:hep-ph/0412366].

[18] C. Delaunay, C. Grojean and J. D. Wells, "Dynamics of Non-renormalizable Electroweak Symmetry Breaking," JHEP 0804 (2008) 029 [arXiv:0711.2511 [hep-ph]].

[19] K. Danzmann and A. Rudiger, "Lisa Technology - Concept, Status, Prospects," Class. Quant. Grav. 20 (2003) S1.

[20] V. Corbin and N. J. Cornish, "Detecting the cosmic gravitational wave background with the 
big bang observer," Class. Quant. Grav. 23 (2006) 2435 arXiv:gr-qc/0512039.

[21] A. Kosowsky, A. Mack and T. Kahniashvili, "Gravitational radiation from cosmological turbulence," Phys. Rev. D 66, 024030 (2002) arXiv:astro-ph/0111483.

[22] A. D. Dolgov, D. Grasso and A. Nicolis, "Relic backgrounds of gravitational waves from cosmic turbulence," Phys. Rev. D 66, 103505 (2002) arXiv:astro-ph/0206461.

[23] C. Caprini and R. Durrer, "Gravitational waves from stochastic relativistic sources: Primordial turbulence and magnetic fields," Phys. Rev. D 74 (2006) 063521 arXiv:astro-ph/0603476].

[24] G. Gogoberidze, T. Kahniashvili and A. Kosowsky, "The spectrum of gravitational radiation from primordial turbulence," Phys. Rev. D 76 (2007) 083002 [arXiv:0705.1733 [astro-ph]].

[25] S. Y. Khlebnikov and I. I. Tkachev, "Relic gravitational waves produced after preheating," Phys. Rev. D 56, 653 (1997) arXiv:hep-ph/9701423.

[26] R. Easther and E. A. Lim, "Stochastic gravitational wave production after inflation," JCAP 0604, 010 (2006) arXiv:astro-ph/0601617.

[27] R. Easther, J. T. Giblin and E. A. Lim, "Gravitational Wave Production At The End Of Inflation," Phys. Rev. Lett. 99, 221301 (2007) arXiv:astro-ph/0612294.

[28] J. Garcia-Bellido and D. G. Figueroa, "A stochastic background of gravitational waves from hybrid preheating," Phys. Rev. Lett. 98 (2007) 061302 [arXiv:astro-ph/0701014].

[29] J. Garcia-Bellido, D. G. Figueroa and A. Sastre, "A Gravitational Wave Background from Reheating after Hybrid Inflation," Phys. Rev. D 77, 043517 (2008) arXiv:0707.0839 [hep$\mathrm{ph}]$.

[30] J. F. Dufaux, A. Bergman, G. N. Felder, L. Kofman and J. P. Uzan, "Theory and Numerics of Gravitational Waves from Preheating after Inflation," Phys. Rev. D 76, 123517 (2007) arXiv:0707.0875 [astro-ph]].

[31] C. Caprini, R. Durrer and G. Servant, "Gravitational wave generation from bubble collisions in first-order phase transitions: an analytic approach," Phys. Rev. D 77, 124015 (2008) arXiv:0711.2593 [astro-ph]].

[32] The effect has been mentioned in refs. [4, 5, 6], but could never be clarified because of numerical uncertainties. 